\title{
Mean-Square Almost Periodic Solutions for Impulsive Stochastic Host-Macroparasite Equation on Time Scales
}

\author{
Pan Wang, ${ }^{1}$ Qingmei Lin, ${ }^{2}$ and Yongkun $\mathrm{Li}^{1}$ \\ ${ }^{1}$ Department of Mathematics, Yunnan University, Kunming, Yunnan 650091, China \\ ${ }^{2}$ School of Mathematics, Wenshan University, Wenshan, Yunnan 663000, China \\ Correspondence should be addressed to Yongkun Li; yklie@ynu.edu.cn
}

Received 26 May 2015; Accepted 26 August 2015

Academic Editor: Eric R. Kaufmann

Copyright (c) 2015 Pan Wang et al. This is an open access article distributed under the Creative Commons Attribution License, which permits unrestricted use, distribution, and reproduction in any medium, provided the original work is properly cited.

\begin{abstract}
We consider an impulsive stochastic host-macroparasite equation on time scales. By use of the Banach fixed point theorem and Gronwall-Bellman's inequality technique on time scales, we obtain the existence and exponential stability of mean-square almost periodic solutions for the host-macroparasite equation on time scales. Finally, we give an example to illustrate the feasibility of our results.
\end{abstract}

\section{Introduction}

Many important human diseases, particularly in tropical and subtropical regions, arise from infection by macroparasites or metazoan organisms. These organisms tend to have much larger generation times and more complex life cycles than microparasites. In life cycles, there are two or more obligatory host species together with the final host (humans). Macroparasitic infections are generally chronic in form and they are more a cause of morbidity than mortality and tend to be persistent in character in areas where they are endemic. The final hosts of parasites are usually humans (the hosts in which the parasite attains reproductive maturity) and they gain entry to the definitive host as a consequence of developmental changes which normally occur before the organism arrives at its preferred site and attains reproductive maturity. Because of this fact, the host-macroparasite system has been attracting the attention of many researchers (see [1-4]). A time delay, therefore, exists between entry to the definitive host and the point when the parasite begins the production of eggs or larvae for transmission to other hosts (see [5-7]). In [7], by means of a continuation theorem in coincidence degree theory, the authors considered the oscillation and global attractivity of the nonlinear delay host-macroparasite model with periodic coefficients.
On the one hand, the theory of impulsive differential equations is now being recognized to not only be richer than the corresponding theory of differential equations without impulses, but also represent a more natural framework for mathematical modelling of many real-world phenomena, such as population dynamical models and neural networks. Since many dynamical processes are characterized by the fact that, at certain moments of time, they undergo abrupt changes of state, with the development of the theory of impulsive differential equations (see $[8,9]$ ), various models of impulsive differential equations have been proposed and studied extensively (see [10-14]). For example, authors of [14] considered the nonlinear impulsive delay host-macroparasite model with periodic coefficients:

$$
\begin{aligned}
& M^{\prime}(t)=-\beta(t) M(t)+\frac{p(t) M(t)}{[q(t)+M(t-n \omega)]^{n+1},}, t \neq t_{k}, \\
& M\left(t_{k}^{+}\right)=\left(1+b_{k}\right) M\left(t_{k}\right), \quad t=t_{k}, \quad k \in \mathbb{N},
\end{aligned}
$$

where $m$ is a positive integer, $q(t)=1 / \delta(t), p(t)=$ $\alpha(t) / \delta^{n+1}(t)$, and $\beta(t)$ are $\omega$-periodic functions. By use of the continuation theorem of coincidence degree, some sufficient 
conditions are obtained for the global attractivity and oscillation of positive periodic solutions.

In fact, both continuous and discrete systems are very important in implementation and applications. But it is troublesome to study the dynamical properties for continuous and discrete systems, respectively. Therefore, it is significant to study that on time scales which can unify the continuous and discrete cases. In [15], the author considered the following host-macroparasite equation on time scales:

$$
x^{\Delta}(t)=-a(t) x(t)+\sum_{i=1}^{m} \frac{b_{i}(t) x\left(t-\tau_{i}(t)\right)}{\left[1+x\left(t-\tau_{i}(t)\right)\right]^{N_{i}+1}} .
$$

By using the contraction principle and Gronwall-Bellman's inequality on time scale, some sufficient conditions are obtained for the existence and exponential stability of almost periodic solutions.

On the other hand, as a matter of fact, population systems are often subject to environmental noise; that is, due to environmental fluctuations, parameters involved in population models are not absolute constants, and they may fluctuate around some average values. Based on these factors, more and more people began to be concerned about stochastic population systems (see [16-20]). Meanwhile, almost periodicity is universal than periodicity, and the mean-square almost periodicity is important in probability for investigating stochastic processes. To the best of our knowledge, there exist few results for mean-square almost periodic solutions for impulsive stochastic process models with delays; one can see some results in [21-23]. However, there exists no result on the existence and uniqueness of mean-square almost periodic solutions for impulsive stochastic host-macroparasite equation on time scales. Motivated by the above, we consider the following impulsive stochastic host-macroparasite equation on time scales:

$$
\begin{aligned}
\Delta x(t)= & {\left[-a(t) x(t)+\sum_{i=1}^{m} \frac{b_{i}(t) x\left(t-\tau_{i}(t)\right)}{\left[1+x\left(t-\tau_{i}(t)\right)\right]^{N_{i}+1}}\right] \Delta t } \\
& +\sum_{i=1}^{m} \delta_{i}(t, x(t-\eta(t))) \Delta w(t), \quad t \neq t_{k}, \\
\widetilde{\Delta} x\left(t_{k}\right)= & x\left(t_{k}+0\right)-x\left(t_{k}-0\right) \\
= & \alpha_{k} x\left(t_{k}\right)+I_{k}\left(x\left(t_{k}\right)\right)+v_{k}, \quad t=t_{k}, k \in \mathbb{N},
\end{aligned}
$$

where $\Delta x(t)$ denotes a $\Delta$-stochastic differential of $x(t), a(t)$, $b_{i}(t), \tau_{i}(t), \eta(t) \in \mathrm{PC}_{\mathrm{rd}}\left(\mathbb{T}, \mathbb{R}^{+}\right), N_{i}>0, \delta_{i}$ is Borel measurable, $i=1,2, \ldots, m, k \in \mathbb{Z},\left\{t_{k}\right\} \in \mathscr{B}=\left\{\left\{t_{k}\right\} \mid t_{k} \in \mathbb{R}, t_{k}<\right.$ $\left.t_{k+1}, \lim _{t \rightarrow \infty} t_{k}=+\infty\right\}, \alpha_{k}, v_{k} \in \mathbb{R}$, and $I_{k} \in C(\mathbb{R}, \mathbb{R})$. Let $(\Omega, \mathbb{F}, \mathbb{P})$ be a complete probability space furnished with a complete family of right continuous increasing sub- $\sigma$ algebras $\left\{\mathscr{F}_{t}\right\}_{t \geq 0}$ satisfying $\mathscr{F}_{t} \subset \mathbb{F} . w(t)$ is a standard Brownian motion over $(\Omega, \mathbb{F}, \mathbb{P})$.

Throughout this paper, we assume the following.

$\left(H_{1}\right) a, b_{i}, \tau_{i}, \eta: \mathbb{T} \rightarrow \mathbb{R}^{+}$are all mean-square almost periodic functions, $a(t) \geq \lambda>0, t-\tau_{i}(t) \in \mathbb{T}, t-\eta(t) \in \mathbb{T}$, for $t \in \mathbb{T}, \delta_{i}: \mathbb{T} \times \mathbb{R} \rightarrow \mathbb{R}^{+}$is mean-square almost periodic in $t$ uniformly for $x \in \mathbb{R}, \delta_{i}(t, 0)=0$, and there exists constant $l_{i}$ such that

$$
\begin{aligned}
& E\left|\delta_{i}(t, x)-\delta_{i}(t, y)\right|^{2} \leq l_{i} E\|x-y\|^{2}, \\
& \quad t \in \mathbb{T}, x, y \in \mathrm{PC}_{\mathrm{rd}}\left(\mathbb{T}, L^{2}(\mathbb{R})\right), i=1,2, \ldots, m .
\end{aligned}
$$

$\left(H_{2}\right)$ The sequences $\alpha_{k}, v_{k}$ are almost periodic in $k$ and the sequences $\left\{I_{k}(x)\right\}$ are almost periodic in $k$ uniformly for $x \in \mathrm{PC}_{\mathrm{rd}}\left(\mathbb{T}, L^{2}(\mathbb{R})\right)$ and there exists constant $L$ such that

$$
\begin{aligned}
E\left|I_{k}(x)-I_{k}(y)\right|^{2} & \leq L E\|x-y\|^{2} \\
& t \in \mathbb{T}, x, y \in \operatorname{PC}_{\mathrm{rd}}\left(\mathbb{\mathbb { T }}, L^{2}(\mathbb{R})\right), i=1,2, \ldots, m .
\end{aligned}
$$

$\left(H_{3}\right)$ The set of sequences $\left\{t_{k}^{j}\right\}, t_{k}^{j}=t_{k+j}-t_{k}, k, j \in \mathbb{N}$, are equipotentially almost periodic and $\inf _{k} t_{k}^{1}=\theta>0$, where $L^{2}(\mathbb{R})$ will be introduced in Section 2 .

For convenience, we denote

$$
\begin{aligned}
& \bar{f}=\sup _{t \in \mathbb{R}}|f(t)|, \\
& \bar{g}=\sup _{(t, x) \in \mathbb{T} \times L^{2}(\mathbb{R})}|g(t, x)|,
\end{aligned}
$$

where $f(t)$ is a mean-square almost periodic function and $g(t, x)$ is a mean-square uniformly almost periodic function on time scales.

Let $\varphi_{0}:\left(t_{0}-\tau, t_{0}\right)_{\mathbb{T}} \rightarrow L^{2}(\mathbb{R})$ be a mean-square almost periodic function. Denote by $x(t)=x\left(t ; t_{0}, \varphi_{0}\right)$ the solution of (3) satisfying the initial conditions:

$$
\begin{aligned}
x\left(t ; t_{0}, \varphi_{0}\right) & =\varphi_{0}(t), \quad t \in\left(t_{0}-\tau, t_{0}\right)_{\mathbb{T}}, \\
x\left(t_{0}^{+} ; t_{0}, \varphi_{0}\right) & =\varphi_{0}\left(t_{0}\right),
\end{aligned}
$$

where $\tau=\sup _{t \in \mathbb{T}}\left\{\max _{i}\left|\tau_{i}(t)\right|,|\eta(t)|\right\}$.

Our main purpose of this paper is to study the existence and exponential stability of mean-square almost periodic solutions to (3) by means of the Banach fixed point theorem and Gronwall-Bellman's inequality technique.

\section{Preliminaries}

In this section, we shall recall some basic definitions and lemmas which are used in what follows.

A time scale $\mathbb{T}$ is an arbitrary nonempty closed subset of the real numbers; the forward and backward jump operators $\sigma, \rho: \mathbb{T} \rightarrow \mathbb{T}$ and the forward graininess $\mu: \mathbb{T} \rightarrow \mathbb{R}^{+}$are defined, respectively, by

$$
\begin{aligned}
& \sigma(t):=\inf \{s \in \mathbb{T}: s>t\}, \\
& \rho(t):=\sup \{s \in \mathbb{T}: s<t\} \\
& \mu(t)=\sigma(t)-t .
\end{aligned}
$$

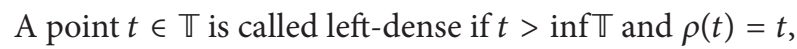
left-scattered if $\rho(t)<t$, right-dense if $t<\sup \mathbb{T}$ and $\sigma(t)=$ $t$, and right-scattered if $\sigma(t)>t$. If $\mathbb{T}$ has a left-scattered 
maximum $m$, then $\mathbb{T}^{k}=\mathbb{T} \backslash\{m\}$; otherwise, $\mathbb{T}^{k}=\mathbb{T}$. If $\mathbb{T}$ has a right-scattered minimum $m$, then $\mathbb{T}_{k}=\mathbb{T} \backslash\{m\}$; otherwise, $\mathbb{T}_{k}=\mathbb{T}$.

A function $f: \mathbb{T} \rightarrow \mathbb{R}$ is right-dense continuous provided it is continuous at right-dense point in $\mathbb{T}$ and its leftside limits exist at left-dense points in $\mathbb{T}$. If $f$ is continuous at each right-dense point and each left-dense point, then $f$ is said to be continuous function on $\mathbb{T}$.

For $y: \mathbb{T} \rightarrow \mathbb{R}$ and $t \in \mathbb{T}^{k}$, we define the delta derivative of $y(t), y^{\Delta}(t)$, to be the number (if it exists) with the property that, for a given $\varepsilon>0$, there exists a neighborhood $U$ of $t$ such that

$$
\left|[y(\sigma(t))-y(s)]-y^{\Delta}(t)[\sigma(t)-s]\right|<\varepsilon|\sigma(t)-s|,
$$

for all $s \in U$.

If $y$ is continuous, then $y$ is right-dense continuous, and if $y$ is delta differentiable at $t$, then $y$ is continuous at $t$.

Let $f$ be right-dense continuous; if $F^{\Delta}(t)=f(t)$, then we define the delta integral by

$$
\int_{r}^{s} f(t) \Delta t=F(s)-F(r), \quad r, s \in \mathbb{T} .
$$

Lemma 1 (see $[24]$ ). Assume $f, g: \mathbb{T} \rightarrow \mathbb{R}$ are delta differentiable at $t \in \mathbb{\mathbb { T }}$; then,

(i) $(f+g)^{\Delta}(t)=f^{\Delta}(t)+g^{\Delta}(t)$;

(ii) $(f g)^{\Delta}(t)=f^{\Delta}(t) g(t)+f^{\sigma}(t) g^{\Delta}(t)=f(t) g^{\Delta}(t)+$ $f^{\Delta}(t) g^{\sigma}(t)$

(iii) if $g(t) g^{\sigma}(t) \neq 0$, then $(f / g)^{\Delta}=\left(f^{\Delta}(t) g(t)-\right.$ $\left.f(t) g^{\Delta}(t)\right) / g(t) g^{\sigma}(t)$;

(iv) if $f$ and $f^{\Delta}$ are continuous, then $\left(\int_{a}^{t} f(t, s) \Delta s\right)^{\Delta}=$ $f(\sigma(t), t)+\int_{a}^{t} f^{\Delta}(t, s) \Delta s$.

A function $p: \mathbb{T} \rightarrow \mathbb{R}$ is called regressive provided $1+$ $\mu(t) p(t) \neq 0$, for all $t \in \mathbb{T}^{k}$. The set of all regressive and $\mathrm{rd}$ continuous functions $p: \mathbb{T} \rightarrow \mathbb{R}$ will be denoted by $\mathscr{R}=$ $\mathscr{R}(\mathbb{T})=\mathscr{R}(\mathbb{T}, \mathbb{R})$. We define the set $\mathscr{R}^{+}=\mathscr{R}^{+}(\mathbb{T}, \mathbb{R})=\{p \in$ $\mathscr{R}: 1+\mu(t) p(t)>0, \forall t \in \mathbb{T}\}$.

If $r \in \mathscr{R}$, then the generalized exponential function $e_{r}$ is defined by

$$
e_{r}(t, s)=\exp \left\{\int_{s}^{t} \xi_{\mu(\tau)}(r(\tau)) \Delta \tau\right\},
$$

for all $s, t \in \mathbb{T}$, with the cylinder transformation

$$
\xi_{h}(z)= \begin{cases}\frac{\log (1+h z)}{h}, & h \neq 0, \\ z, & h=0 .\end{cases}
$$

Let $p, q: \mathbb{T} \rightarrow \mathbb{R}$ be two regressive functions; we define

$$
\begin{aligned}
p \oplus q & =p+q+\mu p q, \\
\ominus p & =-\frac{p}{1+\mu p}, \\
p \ominus q & =p \oplus(\ominus q)=\frac{p-q}{1+\mu q} .
\end{aligned}
$$

Then, the generalized exponential function has the following properties.

Lemma 2 (see [24]). Assume that $p, q: \mathbb{T} \rightarrow \mathbb{R}$ are two regressive functions; then,

(i) $e_{0}(t, s) \equiv 1$ and $e_{p}(t, t) \equiv 1$;

(ii) $e_{p}(\sigma(t), s)=(1+\mu(t) p(t)) e_{p}(t, s)$;

(iii) $e_{p}(t, s)=1 / e_{p}(s, t)=e_{\ominus p}(s, t)$;

(iv) $e_{p}(t, s) e_{p}(s, r)=e_{p}(t, r)$;

(v) $e_{p}(t, s) e_{q}(t, s)=e_{p \oplus q}(t, s)$;

(vi) $e_{p}(t, s) / e_{q}(t, s)=e_{p \ominus q}(t, s)$;

(vii) $\left(1 / e_{p}(t, s)\right)^{\Delta}=-p(t) / e_{p}^{\sigma}(t, s)$

In the following, we will present some basic concepts and results concerning stochastic differential equations on time scales which will be essential to prove our main results. For more details, the reader may want to consult [17].

Definition 3 (see [25]). For a function $f:[0,1]_{\mathbb{T}} \rightarrow \mathbb{R}$, one can define the extension $\tilde{f}:[0,1] \rightarrow \mathbb{R}$ by $\tilde{f}(t)=$ $f\left(\sup [0, t]_{\mathbb{T}}\right)$, for all $t \in[0,1]$.

Let $(\Omega, \mathbb{F}, \mathbb{P})$ be a probability space, and $L^{2}(\mathbb{R})$ stands for a space that consists of all $\mathbb{R}$-valued random variables $x$ with the norm

$$
\mathbb{E}\|x\|^{2}=\int_{w}|x|^{2} \mathrm{~d} \mathbb{P} .
$$

Let $w$ be a standard Wiener process and suppose $\{w(t+h)-$ $w(t): h \geq 0\}$ is independent of $\mathscr{F}_{t}:=\sigma\{w(s): 0 \leq s \leq t\}$, where $\mathbb{F}_{\mathbb{R}}=\left\{\mathscr{F}_{t}: t \in \mathbb{R}\right\}$ is a filtration on $\mathbb{R}$, and with $\sigma\{\cdot\}$, we mean the $\sigma$-algebra generated by $\cdot$. We denote $\Delta$-stochastic integral on $[0,1]_{\mathbb{T}}$, by $\int_{0}^{1} f(t) \Delta w(t)$.

Definition 4 (see [25]). One can say that the random process $f: \mathbb{T} \times \Omega \rightarrow \mathbb{R}$ belongs to the class $L^{2}\left([0,1]_{\mathbb{T}}\right)$ if the following conditions hold:

(i) $f$ is adapted to $\mathbb{F}_{\mathbb{T}}$; that is, $f(t, \cdot)$ is $\mathscr{F}_{t}$-measurable, for all $t \in[0,1]_{\mathbb{T}}$.

(ii) $\mathbb{P}\left(\int_{0}^{1}|f(t, w)|^{2} \Delta t<\infty\right)=1$.

Definition 5 (see [25]). One can say that the random process $f \in L^{2}\left([0,1]_{\mathbb{T}}\right)$ has a $\Delta$-stochastic integral on $[0,1]_{\mathbb{T}}$, provided the corresponding process $\tilde{f}$ has a stochastic integral in the common sense on $[0,1]$ and then one can have $\int_{0}^{1} f(t) \Delta \omega(t)=\int_{0}^{1} \widetilde{f}(t) \mathrm{d} \omega(t)$. 
Lemma 6 (see [25]). The $\Delta$-stochastic integral has the following properties:

(i) If $f_{1}, f_{2} \in L^{2}\left([0,1]_{\mathbb{T}}\right)$ and $c_{1}, c_{2} \in \mathbb{R}$, then

$$
\begin{aligned}
& \int_{0}^{1}\left(c_{1} f_{1}(t)+c_{2} f_{2}(t)\right) \Delta w(t) \\
& \quad=c_{1} \int_{0}^{1} f_{1}(t) \Delta w(t)+c_{2} \int_{0}^{1} f_{2}(t) \Delta w(t) .
\end{aligned}
$$

(ii) If $\mathbb{E}\left(\int_{0}^{1}|f(t)|^{2} \Delta t\right)<+\infty$, then $\mathbb{E}\left(\int_{0}^{1} f(t) \Delta w(t)\right)=0$ and the Itô-isometry holds; that is,

$$
\mathbb{E}\left(\left(\int_{0}^{1} f(t) \Delta w(t)\right)^{2}\right)=\mathbb{E}\left(\int_{0}^{1} f^{2}(t) \Delta t\right) .
$$

Definition 7 (see [25]). If, for all $t_{1}, t_{2} \in[0,1]_{\mathbb{T}}$ and $t_{1}<t_{2}$, such that

$$
X\left(t_{2}\right)-X\left(t_{1}\right)=\int_{t_{1}}^{t_{2}} b(t, X) \Delta t+\int_{t_{1}}^{t_{2}} B(t, X) \Delta w(t),
$$

where $b$ is Lebesgue integrable on $[0,1]_{\mathbb{T}}$ and $B \in L^{2}\left([0,1]_{\mathbb{T}}\right)$, then we say that the process $X$ has a $\Delta$-stochastic differential indicated by the notation

$$
\Delta X(t)=b(t, X) \Delta t+B(t, X) \Delta w(t) .
$$

We consider the impulsive stochastic dynamic system on $\left[t_{0}, a\right]_{\mathbb{T}} \subset \mathbb{T}:$

$$
\begin{array}{r}
\Delta X(t)=b(t) X(t) \Delta t+b(X, t) \Delta t+B(X, t) \Delta w(t), \\
t \neq t_{k},
\end{array}
$$

$$
\widetilde{\Delta} x\left(t_{k}\right)=\alpha_{k} X\left(t_{k}\right)+I_{k}\left(X\left(t_{k}\right)\right), \quad t=t_{k},
$$

with initial value $X\left(t_{0}\right)=X_{0}$, where $X_{0}$ is a random variable, independent of $w(t)$.

Definition 8 (see [23]). One can say that a random process $X(t)$ with $X\left(t_{0}\right)=X_{0}$ is a solution of the impulsive stochastic dynamic system (19) on $\left[t_{0}, a\right]_{\mathbb{\pi}}$ if the following conditions hold:

(i) $X$ is adapted to the filtration $\mathbb{F}$.

(ii) For all $t \in\left[t_{0}, a\right]_{\mathbb{T}}$, we have almost surely

$$
\begin{aligned}
X(t)= & W\left(t, t_{0}\right) X_{0}+\int_{t_{0}}^{t} W(t, \sigma(s)) b(X(s), s) \Delta s \\
& +\int_{t_{0}}^{t} W(t, \sigma(s)) B(X(s), s) \Delta w(s) \\
& +\sum_{t_{0} \leq t_{k}<t} W\left(t, t_{k}\right) I_{k}\left(X\left(t_{k}\right)\right),
\end{aligned}
$$

where $W(t, s)$ is the Cauchy matrix of the following system:

$$
\begin{aligned}
& \Delta X(t)=b(t) X(t) \Delta t, \quad t \neq t_{k}, \\
& \widetilde{\Delta} x\left(t_{k}\right)=\alpha_{k} X\left(t_{k}\right), \quad t=t_{k} .
\end{aligned}
$$

Definition 9 (see [23]). One can say $\varphi: \mathbb{T} \rightarrow L^{2}(\mathbb{R})$ is rd-piecewise continuous with respect to a sequence $\left\{t_{k}\right\} \subset$ $\mathbb{T}$ which satisfies $t_{k}<t_{k+1}, k \in \mathbb{Z}$, if $\varphi(t)$ is continuous on $\left[t_{k}, t_{k+1}\right)_{\mathbb{T}}$ and rd-continuous on $\mathbb{T} \backslash\left\{t_{k}\right\}$. Furthermore, $\left[t_{k}, t_{k+1}\right)_{\mathbb{T}}, k \in \mathbb{Z}$, are called intervals of continuity of the function $\varphi(t)$.

For convenience, $\mathrm{PC}_{\mathrm{rd}}\left(\mathbb{T}, L^{2}(\mathbb{R})\right)$ denotes the set of all piecewise continuous functions with respect to a sequence $\left\{t_{k}\right\}, k \in \mathbb{Z}$. For any integers $k$ and $j$, denote $t_{k}^{j}=t_{k+j}-t_{k}$.

Definition 10 (see [26]). A time scale $\mathbb{T}$ is called an almost periodic time scale if

$$
\Pi=\{\tau \in \mathbb{R}: t \pm \tau \in \mathbb{T}, \forall t \in \mathbb{T}\} \neq\{0\} .
$$

Definition 11 (see [10]). For any $\varepsilon>0$, let $\Gamma_{\varepsilon} \subset \Pi$ be a set of real numbers and $\left\{t_{k}\right\} \subset \mathbb{T}$. One can say $\left\{t_{k}^{j}\right\}, k, j \in \mathbb{Z}$, is equipotentially almost periodic on an almost periodic time scale $\mathbb{T}$ if, for $r \in \Gamma_{\varepsilon} \subset \Pi$, there exists at least one integer $q$ such that

$$
\left|t_{k}^{q}-r\right|<\varepsilon, \quad \forall k \in \mathbb{Z}
$$

Definition 12 (see [23]). Let $\mathbb{T}$ be an almost periodic time scale and assume that $\left\{t_{k}\right\} \subset \mathbb{T}$ satisfying the derived sequence $\left\{t_{k}^{j}\right\}$, $k, j \in \mathbb{Z}$, is equipotentially almost periodic. One can call a stochastic process $\varphi \in \mathrm{PC}_{\mathrm{rd}}\left(\mathbb{T}, L^{2}(\mathbb{R})\right)$ mean-square almost periodic if

(i) for any $\varepsilon>0$, there is a positive number $\delta=\delta(\varepsilon)$ such that if the points $t^{\prime}$ and $t^{\prime \prime}$ belong to the same interval of continuity and $\left|t^{\prime}-t^{\prime \prime}\right|<\delta$, then $\mathbb{E}\left\|\varphi\left(t^{\prime}\right)-\varphi\left(t^{\prime \prime}\right)\right\|^{2}<$ $\varepsilon$;

(ii) for any $\varepsilon>0$, there is relative dense set $\Gamma$ such that if $\tau \in \Gamma$, then $\mathbb{E}\|\varphi(t+\tau)-\varphi(t)\|^{2}<\varepsilon$, for all $t \in \mathbb{T}$, which satisfy the condition $\left|t-\tau_{i}\right|>\varepsilon, i \in \mathbb{Z}$.

Definition 13 (see [23]). Let $\mathbb{T}$ be an almost periodic time scale and assume that $\left\{t_{k}\right\} \subset \mathbb{T}$ satisfying the derived sequence $\left\{t_{k}^{j}\right\}, k, j \in \mathbb{Z}$, is equipotentially almost periodic. One can call a stochastic process $\varphi \in \mathrm{PC}_{\mathrm{rd}}\left(\mathbb{T} \times \Omega, L^{2}(\mathbb{R})\right)$ mean-square almost periodic in $t$ uniformly for $x \in \Omega$ if for any $\varepsilon>0$ and for each compact subset $S$ of $\Omega$ there existed the following:

(i) for any $\varepsilon>0$, there is a positive number $\delta=$ $\delta(\varepsilon, S)$ such that if the points $t^{\prime}$ and $t^{\prime \prime}$ belong to the same interval of continuity and $\left|t^{\prime}-t^{\prime \prime}\right|<\delta$, then $\mathbb{E}\left\|\varphi\left(t^{\prime}, x\right)-\varphi\left(t^{\prime \prime}, x\right)\right\|^{2}<\varepsilon ;$

(ii) for any $\varepsilon>0$, there is relative dense set $\Gamma$ such that if $\tau \in \Gamma$, then $\mathbb{E}\|\varphi(t+\tau, x)-\varphi(t, x)\|^{2}<\varepsilon$, for all $(t, x) \in \mathbb{T} \times \Omega$, which satisfy the condition $\left|t-t_{k}\right|>\varepsilon$, $k \in \mathbb{Z}$

where $\Omega \subset L^{2}(\mathbb{R})$ or $\Omega=L^{2}(\mathbb{R})$. 
Definition 14. The equation (3) is said to be exponentially stable if, for all $c>0$, there exist $\alpha=\alpha(c)>0$ and $K>0$ such that if $\left\|u_{t_{0}}-v_{t_{0}}\right\| \leq c$, then, for all $t \geq t_{0}$,

$$
\begin{gathered}
E\left\|u\left(t ; t_{0}, u_{t_{0}}\right)-v\left(t ; t_{0}, v_{t_{0}}\right)\right\|^{2} \\
<K\left(E\left\|u_{t_{0}}-v_{t_{0}}\right\|^{2}\right) e^{-\alpha\left(t-t_{0}\right)} .
\end{gathered}
$$

In order to study (3), we consider the linear system:

$$
\begin{gathered}
\Delta x(t)=-a(t) x(t) \Delta t, \quad t \neq t_{k}, \\
\widetilde{\Delta} x\left(t_{k}\right)=\alpha_{k} x\left(t_{k}\right), \quad t=t_{k}, k \in \mathbb{N} .
\end{gathered}
$$

Now let us consider the equations

$$
\Delta x(t)=-a(t) x(t) \Delta t, \quad t_{k-1}<t \leq t_{k}
$$

and their solutions

$$
x(t)=x(s) e_{-a}(t, s), \quad t_{k-1}<s \leq t \leq t_{k} .
$$

Then, by [10], the Cauchy matrix of the linear equation (25) is

$$
\begin{aligned}
& W(t, s) \\
& = \begin{cases}e_{-a}(t, s), & t_{k-1}<s \leq t \leq t_{k}, \\
\prod_{i=r}^{k+1}\left(1+\alpha_{i}\right) e_{-a}(t, s), & t_{r-1}<s \leq t_{r}<t_{k}<t \leq t_{k+1}\end{cases}
\end{aligned}
$$

and the solutions of (25) are in the following form:

$$
x\left(t ; t_{0}, \varphi_{0}\right)=W\left(t, t_{0}\right) \varphi_{0}, \quad \varphi_{0} \in \mathbb{R} .
$$

Similar to the proofs of Lemma 3.1 and Lemma 3.2 in [10], one can easily show the following two lemmas, respectively.

Lemma 15. For system (3), let $\left(H_{1}\right)-\left(H_{3}\right)$ hold. Then, for each $\varepsilon>0$, there exist $\varepsilon_{1}>0,0<\varepsilon_{1}<\varepsilon$, a relative dense set $\bar{T} \subset \Pi$ of real numbers and $\mathbb{Q}$, such that the following relations are fulfilled:

(a) $E\|a(t+\tau)-a(t)\|^{2}<\varepsilon, E\left\|b_{i}(t+\tau)-b_{i}(t)\right\|^{2}<\varepsilon, E \| \tau_{i}(t+$ $\tau)-\tau_{i}(t)\left\|^{2}<\varepsilon, E\right\| \xi(t+\tau)-\xi(t)\left\|^{2}<\varepsilon, E\right\| \delta_{i}(t+\tau, x)-$ $\delta_{i}(t, x) \|^{2}<\varepsilon, t \in \mathbb{T}, \tau \in \bar{T}, x \in P C_{r d}\left(\mathbb{T}, L^{2}(\mathbb{R})\right)$, $\left|t-t_{k}\right|>\varepsilon, k \in \mathbb{Z}, i=1,2, \ldots, m ;$

(b) $\left|\alpha_{k+q}-\alpha_{k}\right|<\varepsilon,\left|I_{k+q}(x)-I_{k}(x)\right|<\varepsilon,\left|v_{k+q}-v_{k}\right|<\varepsilon$, $\forall x \in P C_{r d}\left(\mathbb{T}, L^{2}(\mathbb{R})\right), q \in \mathbb{Q}, k \in \mathbb{Z}, i=1,2, \ldots, m ;$

(c) $\left|t_{k}^{q}-\tau\right|<\varepsilon_{1}, q \in \mathbb{Q}, \tau \in \bar{T}, k \in \mathbb{Z}$.

Lemma 16. For (3), let $\left(H_{1}\right)-\left(H_{3}\right)$ hold. Then, the Cauchy matrix $W(t, s)$ of (3) satisfies the inequality

$$
\|W(t, s)\| \leq K e^{-\lambda(t-s)}, \quad t \geq s
$$

and for any $\varepsilon>0, t, s \in \mathbb{T},\left|t-t_{k}\right|>\varepsilon,\left|s-t_{k}\right|>\varepsilon, k \in \mathbb{Z}$, there exists a relatively dense set $\bar{T} \subset \Pi$ of $\varepsilon$-almost periods of the function $a(t)$ and a positive constant $\Gamma$ such that, for $r \in \bar{T}$,

$$
\|W(t+r, s+r)-W(t, s)\| \leq \varepsilon \Gamma e^{-(\lambda / 2)(t-s)}, \quad t \geq s .
$$

Definition 17. The equation (3) is said to be exponentially stable if, for all $c>0$, there exist $\alpha=\alpha(c)>0$ and $M>0$ such that if $\left\|u_{t_{0}}-v_{t_{0}}\right\| \leq c$, then, for all $t \geq t_{0}$,

$$
\begin{aligned}
E \| & u\left(t ; t_{0}, u_{t_{0}}\right)-v\left(t ; t_{0}, v_{t_{0}}\right) \|^{2} \\
& <M\left(E\left\|u_{t_{0}}-v_{t_{0}}\right\|^{2}\right) e_{\ominus \alpha}\left(t, t_{0}\right) .
\end{aligned}
$$

\section{Existence of Almost Periodic Solution}

In this section, we will study the existence and exponential stability of mean-square almost periodic solutions of (3) by using the Banach fixed point theorem and GronwallBellman's inequality technique on time scales.

Theorem 18. Assume that $\left(H_{1}\right)-\left(H_{3}\right)$ hold and there exist two positive constants $d, N$ such that the following conditions hold.

$\left(H_{4}\right) \gamma<1$, where

$$
\begin{aligned}
\gamma & =\left\{\frac{3 K^{2}(1+\bar{\mu} \lambda)^{2}}{\lambda^{2}}\left[\left(\sum_{i=1}^{m} \overline{b_{i}} M_{i}\right)^{2}+\left(\sum_{i=1}^{m} \overline{\delta_{i}}\right)^{2}\right]\right. \\
& \left.+3\left(\frac{K L}{1-e^{-\lambda \theta}}\right)^{2}\right\}, \\
M_{i} & =\frac{1}{N_{i}\left(1+1 / N_{i}\right)^{1+N_{i}}}, \quad i=1,2, \ldots, m .
\end{aligned}
$$

$\left(H_{5}\right)(\ominus \lambda) \oplus p<0$, where

$$
p=\frac{4 K^{2}(1+\lambda \bar{\mu})^{2}}{\lambda}\left[\left(\sum_{i=1}^{m} \bar{b}_{i}\right)^{2}+\left(\sum_{i=1}^{m} l_{i}\right)^{2}\right]
$$

Then,

(1) There exists a unique square-mean almost periodic solution $x(t)$ of (3) in the region $B^{*}=\{\varphi \mid \varphi \in$ $\left.P C_{r d}\left(\mathbb{T}, L^{2}(\mathbb{R})\right), E\|\varphi(t)\|^{2} \leq d^{2}, t \in \mathbb{T}\right\}$.

(2) The solution $x(t)$ in the region $B^{*}$ is exponentially stable.

Proof. We define an operator $T$ in $B^{*}$ as

$$
\begin{aligned}
T \varphi= & \int_{-\infty}^{t} W(t, \sigma(s)) S_{\varphi}(s) \Delta s \\
& +\int_{-\infty}^{t} W(t, \sigma(s)) L_{\varphi}(s) \Delta w(s) \\
& +\sum_{t_{k}<t} W\left(t, t_{k}\right) h_{\varphi}^{k},
\end{aligned}
$$


where

$$
\begin{aligned}
S_{\varphi}(s) & =\sum_{i=1}^{m} \frac{b_{i}(s) \varphi\left(s-\tau_{i}(s)\right)}{\left[1+\varphi\left(s-\tau_{i}(s)\right)\right]^{N_{i}+1}}, \\
L_{\varphi}(s) & =\sum_{i=1}^{m} \delta_{i}(s, x(s-\eta(s))), \\
h_{\varphi}^{k} & =I_{k}\left(\varphi\left(t_{k}\right)\right)+v_{k}, \quad k \in \mathbb{Z} .
\end{aligned}
$$

For any $\varphi \in B^{*}$, by means of the proof of Theorem 1 in [19], we have

$$
\begin{aligned}
\left\|S_{\varphi}\right\| & =\sup _{s \in \mathbb{T}}\left|\sum_{i=1}^{m} \frac{b_{i}(s) \varphi\left(s-\tau_{i}(s)\right)}{\left[1+\varphi\left(s-\tau_{i}(s)\right)\right]^{N_{i}+1}}\right| \\
& \leq \sup _{s \in \mathbb{T}}\left|\sum_{i=1}^{m} \frac{\overline{b_{i}} \varphi\left(s-\tau_{i}(s)\right)}{\left[1+\varphi\left(s-\tau_{i}(s)\right)\right]^{N_{i}+1}}\right| \leq \sum_{i=1}^{m} \bar{b}_{i} M_{i} .
\end{aligned}
$$

Firstly, we will show that $T$ is a self-mapping from $B^{*}$ to $B^{*}$. In fact, for any $\varphi \in B^{*}$, by use of (37), we have

\section{$\mathbb{E}\|T \varphi\|^{2}$}

$$
\begin{aligned}
\leq & 3 \mathbb{E}\left(\int_{-\infty}^{t}\|W(t, \sigma(s))\|\left\|S_{\varphi}(s)\right\| \Delta s\right)^{2} \\
& +3 \mathbb{E}\left(\left\|\int_{-\infty}^{t} W(t, \sigma(s)) L_{\varphi}(s) \Delta w(s)\right\|\right)^{2} \\
& +3 \mathbb{E}\left(\sum_{t_{k}<t}\left\|W\left(t, t_{k}\right)\right\|\left\|h_{\varphi}^{k}\right\|\right)^{2} \\
\leq & \frac{3 K^{2}(1+\bar{\mu} \lambda)^{2}}{\lambda^{2}}\left[\left(\sum_{i=1}^{m} \overline{b_{i}} M_{i}\right)^{2}+\left(\sum_{i=1}^{m} \overline{\delta_{i}}\right)^{2}\right] \\
& +3\left(\frac{K}{1-e^{-\lambda \theta}} \sup _{k \in \mathbb{Z}}\left(\overline{I_{k}}+\left|v_{k}\right|\right)\right)^{2}:=d^{2} .
\end{aligned}
$$

On the other hand, let $\tau \in \bar{T}$ and $q \in \mathbb{Q}$, where the set $\bar{T}$ is determined in Lemma 15 . Then,

$$
\begin{aligned}
& \|T \varphi(t+\tau)-T \varphi(t)\| \\
& \leq \| \int_{-\infty}^{t}[W(t+\tau, \sigma(s+\tau))-W(t, \sigma(s))] \\
& \cdot S_{\varphi}(s+\tau) \Delta s+\int_{-\infty}^{t} W(t, \sigma(s)) \\
& \cdot\left[S_{\varphi}(s+\tau)-S_{\varphi}(s)\right] \Delta s \\
& +\int_{-\infty}^{t}[W(t+\tau, \sigma(s+\tau))-W(t, \sigma(s))] \\
& \cdot L_{\varphi}(s+\tau) \Delta w(s)+\int_{-\infty}^{t} W(t, \sigma(s))
\end{aligned}
$$

$$
\begin{aligned}
& \cdot\left[L_{\varphi}(s+\tau)-L_{\varphi}(s)\right] \Delta w(s)+\sum_{t_{k}<t+\tau} W\left(t+\tau, t_{k}\right) \\
& \cdot h_{\varphi}^{k}-\sum_{t_{k}<t} W\left(t, t_{k}\right) h_{\varphi}^{k}\|\leq\| \sum_{i=1}^{m} \bar{b}_{i} M_{i} \\
& \cdot \int_{-\infty}^{t}[W(t+\tau, \sigma(s+\tau))-W(t, \sigma(s))] \Delta s \\
& +\int_{-\infty}^{t} W(t, \sigma(s))\left[S_{\varphi}(s+\tau)-S_{\varphi}(s)\right] \Delta s+\sum_{i=1}^{m} \overline{\delta_{i}} \\
& \cdot \int_{-\infty}^{t}[W(t+\tau, \sigma(s+\tau))-W(t, \sigma(s))] \Delta w(s) \\
& +\int_{-\infty}^{t} W(t, \sigma(s))\left[L_{\varphi}(s+\tau)-L_{\varphi}(s)\right] \Delta w(s) \\
& +\sum_{t_{k}<t}\left[W\left(t+\tau, t_{k+q}\right)-W\left(t, t_{k}\right)\right] h_{\varphi}^{k+q} \\
& +\sum_{t_{k}<t} W\left(t, t_{k}\right)\left(h_{\varphi}^{k+q}-h_{\varphi}^{k}\right)\|=\| J_{1}+J_{2}+J_{3} \|,
\end{aligned}
$$

where

$$
\begin{aligned}
J_{1} & =\sum_{i=1}^{m} \overline{b_{i}} M_{i} \\
& \cdot \int_{-\infty}^{t}[W(t+\tau, \sigma(s+\tau))-W(t, \sigma(s))] \Delta s \\
& +\int_{-\infty}^{t} W(t, \sigma(s))\left[S_{\varphi}(s+\tau)-S_{\varphi}(s)\right] \Delta s, \\
J_{2}= & \sum_{i=1}^{m} \overline{\delta_{i}} \\
& \cdot \int_{-\infty}^{t}[W(t+\tau, \sigma(s+\tau))-W(t, \sigma(s))] \Delta w(s) \\
& +\int_{-\infty}^{t} W(t, \sigma(s))\left[L_{\varphi}(s+\tau)-L_{\varphi}(s)\right] \Delta w(s), \\
J_{3}= & \sum_{t_{k}<t}\left[W\left(t+\tau, t_{k+q}\right)-W\left(t, t_{k}\right)\right] h_{\varphi}^{k+q} \\
& +\sum_{t_{k}<t} W\left(t, t_{k}\right)\left(h_{\varphi}^{k+q}-h_{\varphi}^{k}\right) .
\end{aligned}
$$

Since $(a+b+c)^{2} \leq 3 a^{2}+3 b^{2}+3 c^{2}$, it follows that

$$
\begin{aligned}
& \mathbb{E}\|T \varphi(t+\tau)-T \varphi(t)\|^{2} \\
& \quad \leq 3 \mathbb{E}\left\|J_{1}\right\|^{2}+3 \mathbb{E}\left\|J_{2}\right\|^{2}+3 \mathbb{E}\left\|J_{3}\right\|^{2} .
\end{aligned}
$$

By conditions $\left(H_{1}\right)-\left(H_{3}\right)$ and Lemmas 15 and 16, we can obtain that

$$
\mathbb{E}\|T \varphi(t+\tau)-T \varphi(t)\|^{2} \leq 3\left(c_{1}^{2}+c_{2}^{2}+c_{3}^{2}\right) \varepsilon,
$$


where

$$
\begin{aligned}
& c_{1}=\frac{\Gamma(2+\bar{\mu} \lambda)}{\lambda} \sum_{i=1}^{m} \overline{b_{i}} M_{i}+\frac{K(1+\bar{\mu} \lambda)}{\lambda}, \\
& c_{2}=\frac{\Gamma(2+\bar{\mu} \lambda)}{\lambda} \sum_{i=1}^{m} \overline{\delta_{i}}+\frac{K(1+\bar{\mu} \lambda)}{\lambda}, \\
& c_{3}=\frac{\Gamma}{1-e^{-(\lambda / 2) \theta}} \sup _{k \in \mathbb{Z}}\left(\overline{I_{k}}+\left|v_{k}\right|\right)+\frac{K}{1-e^{-\lambda \theta}} .
\end{aligned}
$$

Consequently, by (39) and (42), we obtain that $T \varphi \in B^{*}$.

Next, we prove that the mapping $T$ is a contraction mapping of $B^{*}$. In fact, in view of $\left(H_{1}\right)-\left(H_{3}\right)$, for any $\varphi, \psi \in$ $B^{*}$, we can easily obtain

$$
\begin{gathered}
\mathbb{E}\left\|S_{\varphi}(s)-S_{\psi}(s)\right\|^{2} \leq\left(\sum_{i=1}^{m} \overline{b_{i}}\right)^{2} \mathbb{E}\|\varphi-\psi\|^{2}, \\
\mathbb{E}\left\|L_{\varphi}(s)-L_{\psi}(s)\right\|^{2} \leq\left(\sum_{i=1}^{m} l_{i}\right)^{2} \mathbb{E}\|\varphi-\psi\|^{2} .
\end{gathered}
$$

Notice that

$$
\begin{aligned}
& E\|T(\varphi)-T(\psi)\|^{2} \\
& \leq 3 E\left\|\int_{-\infty}^{t} W(t, \sigma(s))\left[S_{\varphi}(s)-S_{\psi}(s)\right] \Delta s\right\|^{2} \\
& +3 E\left\|\int_{-\infty}^{t} W(t, \sigma(s))\left[L_{\varphi}(s)-L_{\psi}(s)\right] \Delta w(s)\right\|^{2} \\
& +3 E\left\|\sum_{t_{k}<t} W\left(t, t_{k}\right)\left[h_{\varphi}^{k}-h_{\psi}^{k}\right]\right\|^{2} \\
& \quad \leq\left\{\frac{3 K^{2}(1+\bar{\mu} \lambda)^{2}}{\lambda^{2}}\left[\left(\sum_{i=1}^{m} \overline{b_{i}}\right)^{2}+\left(\sum_{i=1}^{m} l_{i}\right)^{2}\right]\right. \\
& \left.\quad+3\left(\frac{K L}{1-e^{-\lambda \theta}}\right)^{2}\right\} \mathbb{E}\|\varphi-\psi\|^{2}=\gamma \mathbb{E}\|\varphi-\psi\|^{2} .
\end{aligned}
$$

This implies that the mapping $T$ is a contraction mapping. Hence, there exists a unique mean-square almost periodic solution of (3) by the Banach fixed point theorem. Suppose that $y(t)$ is an arbitrary solution of (3) with initial conditions:

$$
\begin{aligned}
y\left(t ; t_{0}, \phi_{0}\right) & =\phi_{0}(t), \quad t \in\left(t_{0}-\bar{\tau}, t_{0}\right), \\
y\left(t_{0}^{+} ; t_{0}, \phi_{0}\right) & =\phi_{0}\left(t_{0}\right) .
\end{aligned}
$$

Since every solution of system (3) can be represented as

$$
\begin{aligned}
x(t)= & W\left(t, t_{0}\right) \varphi_{0}+\int_{t_{0}}^{t} W(t, \sigma(s)) S_{x}(s) \Delta s \\
& +\int_{t_{0}}^{t} W(t, \sigma(s)) L_{x}(s) \Delta w(s) \\
& +\sum_{t_{0} \leq t_{k}<t} W\left(t, t_{k}\right) h_{x}^{k},
\end{aligned}
$$

we obtain that

$$
\begin{aligned}
y(t)- & x(t) \\
= & W\left(t, t_{0}\right)\left(\phi_{0}-\varphi_{0}\right) \\
& +\int_{t_{0}}^{t} W(t, \sigma(s))\left(S_{y}(s)-S_{x}(s)\right) \Delta s \\
& +\int_{t_{0}}^{t} W(t, \sigma(s))\left(L_{y}(s)-L_{x}(s)\right) \Delta w(s) \\
& +\sum_{t_{0} \leq t_{k}<t} W\left(t, t_{k}\right)\left(h_{y}^{k}-h_{x}^{k}\right) .
\end{aligned}
$$

Since $(a+b+c+d)^{2} \leq 4 a^{2}+4 b^{2}+4 c^{2}+4 d^{2}$, it follows that

$$
\begin{aligned}
& \mathbb{E}\|y(t)-x(t)\|^{2} \leq 4 K^{2} e^{-\lambda\left(t-t_{0}\right)} \mathbb{E}\left\|\phi_{0}-\varphi_{0}\right\|^{2} \\
& +4 K^{2}\left[\left(\sum_{i=1}^{m} \overline{b_{i}}\right)^{2}+\left(\sum_{i=1}^{m} l_{i}\right)^{2}\right]\left(\int_{-\infty}^{t} e^{-\lambda(t-\sigma(s))} \Delta s\right) \\
& \cdot\left(\int_{t_{0}}^{t} e^{-\lambda(t-\sigma(s))} \mathbb{E}\|y(s)-x(s)\|^{2} \Delta s\right)+4(K L)^{2} \\
& \cdot\left(\sum_{-\infty \leq t_{k}<t} e^{-\lambda\left(t-t_{k}\right)}\right) \\
& \cdot \sum_{t_{0} \leq t_{k}<t} e^{-\lambda\left(t-t_{k}\right)} \mathbb{E}\left\|y\left(t_{k}\right)-x\left(t_{k}\right)\right\|^{2} \\
& \leq 4 K^{2} e_{\ominus \lambda}\left(t, t_{0}\right) \mathbb{E}\left\|\phi_{0}-\varphi_{0}\right\|^{2} \\
& +\frac{4 K^{2}(1+\lambda \bar{\mu})}{\lambda}\left[\left(\sum_{i=1}^{m} \bar{b}_{i}\right)^{2}+\left(\sum_{i=1}^{m} l_{i}\right)^{2}\right]
\end{aligned}
$$

$$
\begin{aligned}
& \cdot\left(\int_{t_{0}}^{t} e_{\ominus \lambda}(t, \sigma(s)) \mathbb{E}\|y(s)-x(s)\|^{2} \Delta s\right) \\
& +\frac{4(K L)^{2}}{1-e^{-\lambda \theta}} \sum_{t_{0} \leq t_{k}<t} e_{\ominus \lambda}\left(t, t_{k}\right) \mathbb{E}\left\|y\left(t_{k}\right)-x\left(t_{k}\right)\right\|^{2} \\
& \leq 4 K^{2} e_{\ominus \lambda}\left(t, t_{0}\right) \mathbb{E}\left\|\phi_{0}-\varphi_{0}\right\|^{2}
\end{aligned}
$$$$
+\frac{4 K^{2}(1+\lambda \bar{\mu})^{2}}{\lambda}\left[\left(\sum_{i=1}^{m} \bar{b}_{i}\right)^{2}+\left(\sum_{i=1}^{m} l_{i}\right)^{2}\right]
$$$$
\cdot\left(\int_{t_{0}}^{t} e_{\ominus \lambda}(t, s) \mathbb{E}\|y(s)-x(s)\|^{2} \Delta s\right)+\frac{4(K L)^{2}}{1-e^{-\lambda \theta}}
$$$$
\cdot \sum_{t_{0} \leq t_{k}<t} e_{\ominus \lambda}\left(t, t_{k}\right) \mathbb{E}\left\|y\left(t_{k}\right)-x\left(t_{k}\right)\right\|^{2}
$$ 
Let $\mathbb{E}\|u(t)\|^{2}=\mathbb{E}\|y(t)-x(t)\|^{2} e_{\lambda}\left(t, t_{0}\right)$; then,

$$
\begin{aligned}
& \mathbb{E}\|u(t)\|^{2} \leq 4 \mathbb{E}\left\|u\left(t_{0}\right)\right\|^{2} \\
& +\frac{4 K^{2}(1+\lambda \bar{\mu})^{2}}{\lambda}\left[\left(\sum_{i=1}^{m} \overline{b_{i}}\right)^{2}+\left(\sum_{i=1}^{m} l_{i}\right)^{2}\right] \\
& \cdot \int_{t_{0}}^{t} \mathbb{E}\|u(s)\|^{2} \Delta s+\frac{4(K L)^{2}}{1-e^{-\lambda \theta}} \sum_{t_{0} \leq t_{k}<t} \mathbb{E}\left\|u\left(t_{k}\right)\right\|^{2} .
\end{aligned}
$$

From Gronwall-Bellman's inequality on time scales, one has

$$
\begin{aligned}
& \mathbb{E}\|u(t)\|^{2} \\
& \quad \leq 4 K^{2} \mathbb{E}\left\|u\left(t_{0}\right)\right\|^{2} \prod_{t_{0}<t_{k}<t}\left(1+\frac{4(K L)^{2}}{1-e^{-\lambda \theta}}\right) e_{p}\left(t, t_{0}\right)
\end{aligned}
$$

that is,

$$
\begin{aligned}
& \mathbb{E}\|y(t)-x(t)\|^{2} \\
& \quad \leq \mathbb{E}\left\|\phi_{0}-\varphi_{0}\right\|^{2} \prod_{t_{0}<t_{k}<t}\left(1+\frac{4(K L)^{2}}{1-e^{-\lambda \theta}}\right) e_{(\ominus \lambda) \oplus p}\left(t, t_{0}\right),
\end{aligned}
$$

where

$$
p=\frac{4 K^{2}(1+\lambda \bar{\mu})^{2}}{\lambda}\left[\left(\sum_{i=1}^{m} \bar{b}_{i}\right)^{2}+\left(\sum_{i=1}^{m} l_{i}\right)^{2}\right]
$$

Thus, by condition $\left(\mathrm{H}_{5}\right)$ of the theorem, the mean-quare almost periodic solution of (3) is exponentially stable. This completes the proof.

Remark 19. According to the conditions of Theorem 18, we can find that the uniqueness and exponential stability of the mean-square almost periodic solution for the impulsive stochastic host-macroparasite equation on time scales are independent of the magnitude of delays but are dependent on the magnitude of noise and impulse.

Remark 20. It is the first time that the sufficient conditions for the existence and exponential stability of piecewise meansquare almost periodic solutions for the impulsive stochastic host-macroparasite equation on time scales are investigated. The obtained results are essentially new. Without considering impulsive and stochastic effects on (3), then (3) reduce to (2).

\section{An Example}

Consider the following impulsive stochastic host-macroparasite equation on time scales:

$\Delta x(t)$

$$
\begin{gathered}
=\left[-a(t) x(t)+\sum_{i=1}^{m} \frac{b_{i}(t) x\left(t-\tau_{i}(t)\right)}{\left[1+x\left(t-\tau_{i}(t)\right)\right]^{N_{i}+1}}\right] \Delta t \\
+\sum_{i=1}^{m} \delta_{i}(t, x(t-\eta(t))) \Delta \omega(t), \quad t \neq t_{k},
\end{gathered}
$$

$$
\begin{aligned}
& \widetilde{\Delta} x\left(t_{k}\right)=x\left(t_{k}+0\right)-x\left(t_{k}-0\right) \\
& \quad=\alpha_{k} x\left(t_{k}\right)+I_{k}\left(x\left(t_{k}\right)\right)+v_{k}, \quad t=t_{k}, k \in \mathbb{N},
\end{aligned}
$$

where $i=2, t_{k}=k, k \in \mathbb{N}$, and

$$
\begin{aligned}
a(t) & =8+\cos \sqrt{2} t, \\
b_{1}(t) & =0.03(1+|\cos \sqrt{3} t|), \\
b_{2}(t) & =0.05(1+|\sin \sqrt{2} t|), \\
\delta_{1}(t, x) & =0.04|\sin \sqrt{5} t x|, \\
\delta_{2}(t, x) & =0.02|\sin \sqrt{2} t x|, \\
\alpha_{k} & =-0.06|\cos k|, \\
I_{k}(x) & =0.06|\sin k x|, \\
v_{k} & =0.07, \\
N_{1} & =1, \\
N_{2} & =2 .
\end{aligned}
$$

By calculating, we have

$$
\begin{aligned}
\bar{a} & =9, \\
\overline{b_{1}} & =0.06, \\
\overline{b_{2}} & =0.1, \\
\overline{\delta_{1}} & =l_{1}=0.04, \\
\overline{\delta_{2}} & =l_{2}=0.02, \\
L & =0.06, \\
M_{1} & =0.25, \\
M_{2} & =\frac{4}{27} .
\end{aligned}
$$


It is easy to see that $K=\theta=1, \lambda=5$; thus,

$$
\begin{aligned}
& \frac{3 K^{2}(1+\bar{\mu} \lambda)^{2}}{\lambda^{2}}\left[\left(\sum_{i=1}^{m} \bar{b}_{i} M_{i}\right)^{2}+\left(\sum_{i=1}^{m} \bar{\delta}_{i}\right)^{2}\right] \\
& +3\left(\frac{K L}{1-e^{-\lambda \theta}}\right)^{2} \approx 0.046<1, \\
& p-\lambda=\frac{4 K^{2}(1+\lambda \bar{\mu})^{2}}{\lambda}\left[\left(\sum_{i=1}^{m} \bar{b}_{i}\right)^{2}+\left(\sum_{i=1}^{m} l_{i}\right)^{2}\right]-\lambda \\
& \approx 0.841-5=-4.159<0 .
\end{aligned}
$$

Hence, $(\ominus \lambda) \oplus p<0$. According to Theorem 18, the impulsive stochastic equation (54) has a unique piecewise mean-square almost periodic solution which is exponentially stable in the region $B^{*}$, where

$$
\begin{aligned}
B^{*} & =\left\{\varphi \mid \varphi \in \mathrm{PC}_{\mathrm{rd}}\left(\mathbb{T}, L^{2}(\mathbb{R})\right), E\|\varphi(t)\|^{2} \leq 1, t\right. \\
& \in \mathbb{T}\} .
\end{aligned}
$$

\section{Conclusion}

In this paper, we have investigated the host-macroparasite equation with impulsive and stochastic effects on time scales. By employing the Banach fixed point theorem, some stochastic analysis techniques, and Gronwall-Bellman's inequality technique on time scales, we have obtained some sufficient conditions ensuring the existence and exponential stability of mean-square almost periodic solutions for impulsive hostmacroparasite equation on time scales. These sufficient conditions can be easily checked by simple algebraic methods. An example has been given to demonstrate the effectiveness of the presented results. The methods used in paper can be applied to study square-mean almost periodic problems of many other types of impulsive stochastic models on time scales.

\section{Conflict of Interests}

The authors declare that there is no conflict of interests regarding the publication of this paper.

\section{Acknowledgment}

This work is supported by the National Natural Sciences Foundation of People's Republic of China under Grant 11361072.

\section{References}

[1] V. A. Kostitzin, Symbiose, Parasitisme et Évolution, Hermann \& Cie, Paris, France, 1943.

[2] N. G. Hairston, "On the mathematical analysis of schistosome populations," Bulletin of the World Health Organization, vol. 33, no. 1, pp. 45-62, 1965.
[3] G. M. Tallis and M. Leyton, "A stochastic approach to the study of parasite populations," Journal of Theoretical Biology, vol. 13, pp. 251-260, 1966.

[4] M. K. Leyton, "Stochastic models in populations of helminthic parasites in the definitive host, II: sexual mating functions," Mathematical Biosciences, vol. 3, no. 1-2, pp. 413-419, 1968.

[5] R. May and R. M. Anderson, Infectious Diseases of Humans: Dynamics and Control, Oxford Science Publications, 1995.

[6] N. MacDonald, Biological Delay Systems: Linear Stability Theory, vol. 8 of Cambridge Studies in Mathematical Biology, Cambridge University Press, Cambridge, UK, 1989.

[7] R. P. Agarwal, D. O’Regan, and S. H. Saker, "Oscillation and global attractivity in a delay periodic host macroparasite model," Applied Mathematics and Computation, vol. 196, no. 1, pp. 340-352, 2008.

[8] V. Lakshmikantham, D. D. Bainov, and P. S. Simeonov, Theory of Impulsive Differential Equations, World Scientific, Singapore, 1989.

[9] A. M. Samoǐlenko and N. A. Perestyuk, Impulsive Differential Equations, Singapore, World Scientific Publishing, 1995.

[10] C. Wang, "Almost periodic solutions of impulsive BAM neural networks with variable delays on time scales," Communications in Nonlinear Science and Numerical Simulation, vol. 19, no. 8, pp. 2828-2842, 2014.

[11] J. O. Alzabut, "Almost periodic solutions for an impulsive delay Nicholson's blowflies model," Journal of Computational and Applied Mathematics, vol. 234, no. 1, pp. 233-239, 2010.

[12] L. Wang and Z. Liu, "Analysis of a periodic impulsive predatorprey system with disease in the prey," Journal of Applied Mathematics, vol. 2013, Article ID 656920, 13 pages, 2013.

[13] G. T. Stamov, "On the existence of almost periodic solutions for the impulsive Lasota-Wazewska model," Applied Mathematics Letters, vol. 2, no. 4, pp. 516-520, 2009.

[14] S. H. Saker and J. O. Alzabut, "Periodic solutions, global attractivity and oscillation of an impulsive delay host-macroparasite model," Mathematical and Computer Modelling, vol. 45, no. 5-6, pp. 531-543, 2007.

[15] Z. J. Yao, "Existence and global exponential stability of an almost periodic solution for a host-macroparasite equation on time scales," Advances in Difference Equations, vol. 2015, article 41, 2015.

[16] H. Qiu, J. Lv, and K. Wang, "Two types of permanence of a stochastic mutualism model," Advances in Difference Equations, vol. 2013, article 37, 2013.

[17] D. Jiang, N. Shi, and X. Li, "Global stability and stochastic permanence of a non-autonomous logistic equation with random perturbation," Journal of Mathematical Analysis and Applications, vol. 340, no. 1, pp. 588-597, 2008.

[18] X. Li and X. Mao, "Population dynamical behavior of nonautonomous Lotka-Volterra competitive system with random perturbation," Discrete and Continuous Dynamical Systems Series A, vol. 24, no. 2, pp. 523-545, 2009.

[19] M. Liu, K. Wang, and Y. Wang, "Long term behaviors of stochastic single-species growth models in a polluted environment II," Applied Mathematical Modelling, vol. 35, no. 9, pp. 4438-4448, 2011.

[20] M. Liu and K. Wang, "Persistence and extinction in stochastic non-autonomous logistic systems," Journal of Mathematical Analysis and Applications, vol. 375, no. 2, pp. 443-457, 2011.

[21] H. Zhou, Z. Zhou, and Z. Qiao, "Mean-square almost periodic solution for impulsive stochastic Nicholson's blowflies model 
with delays," Applied Mathematics and Computation, vol. 219, no. 11, pp. 5943-5948, 2013.

[22] R. Zhang, N. Ding, and L. Wang, "Mean square almost periodic solutions for impulsive stochastic differential equations with delays," Journal of Applied Mathematics, vol. 2012, Article ID 414320, 14 pages, 2012.

[23] C. Wang, "Existence and exponential stability of piecewise mean-square almost periodic solutions for impulsive stochastic Nicholson's blowflies model on time scales," Applied Mathematics and Computation, vol. 248, pp. 101-112, 2014.

[24] M. Bohner and A. Peterson, Advances in Dynamic Equations on Time Scales, Birkhäuser, Boston, Mass, USA, 2003.

[25] M. Bohner, O. M. Stanzhytskyi, and A. O. Bratochkina, "Stochastic dynamic equations on general time scales," Electronic Journal of Differential Equations, no. 57, pp. 1-15, 2013.

[26] Y. K. Li and C. Wang, "Uniformly almost periodic functions and almost periodic solutions to dynamic equations on time scales," Abstract and Applied Analysis, vol. 2011, Article ID 341520, 22 pages, 2011. 


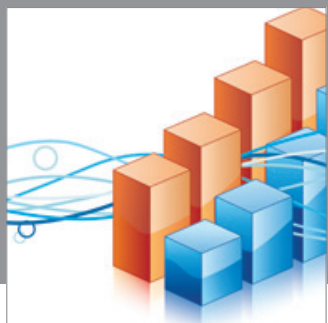

Advances in

Operations Research

mansans

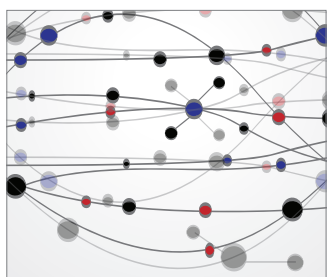

The Scientific World Journal
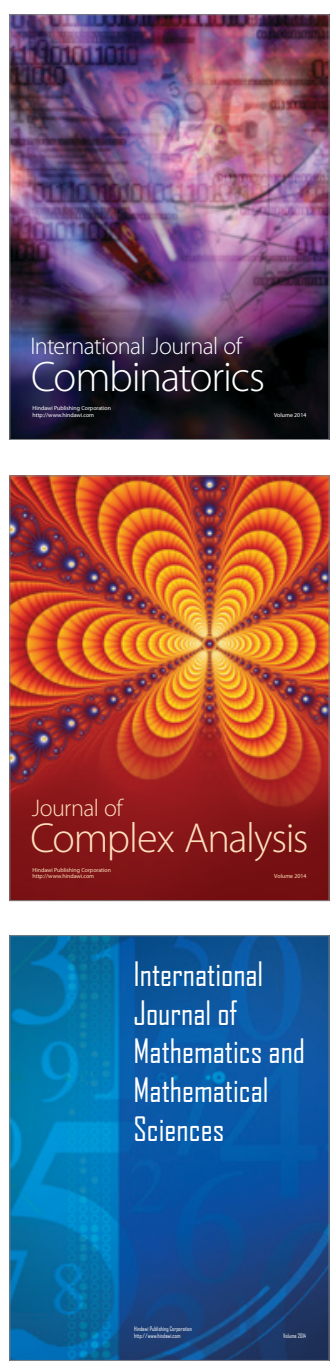
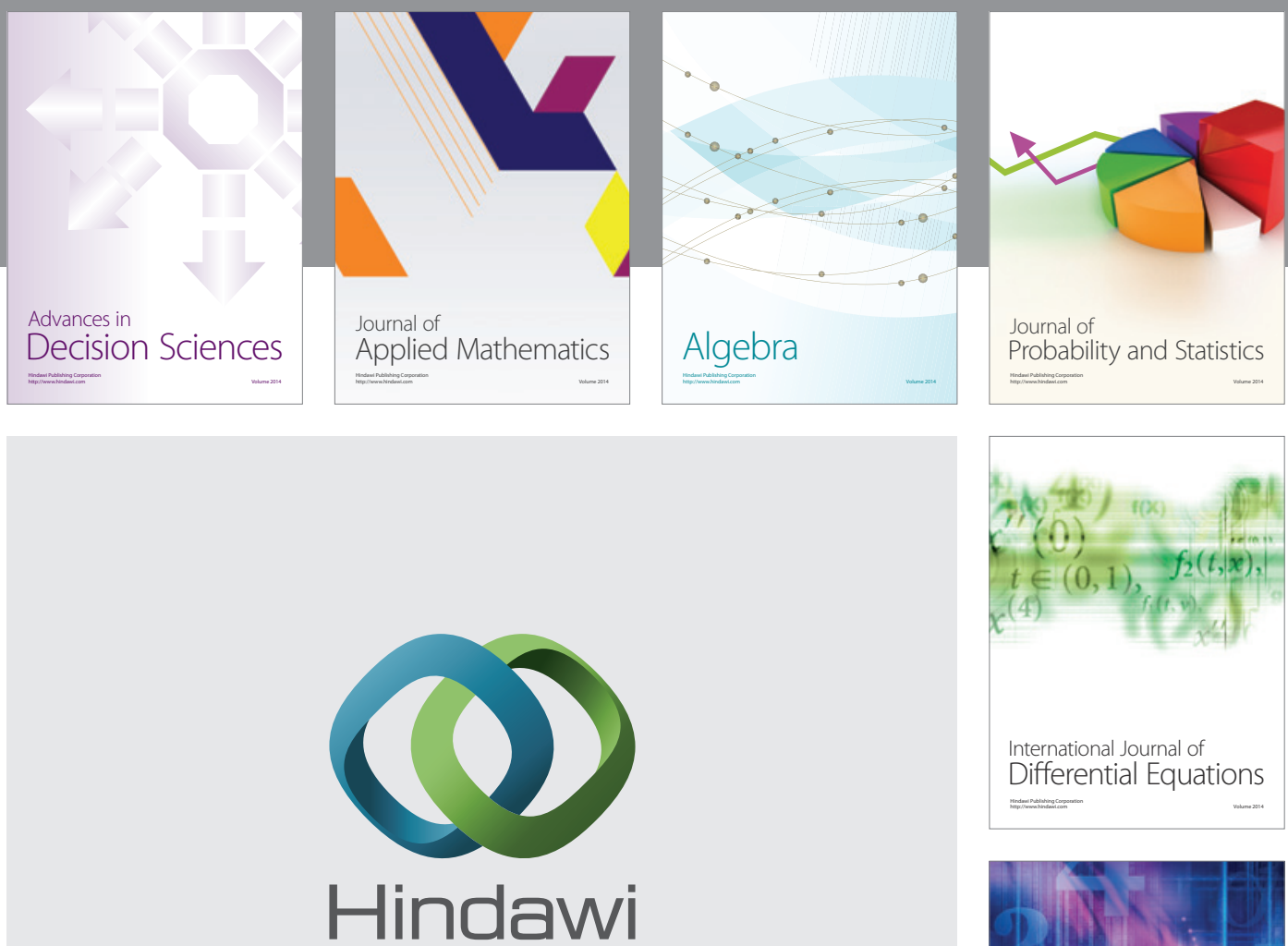

Submit your manuscripts at http://www.hindawi.com
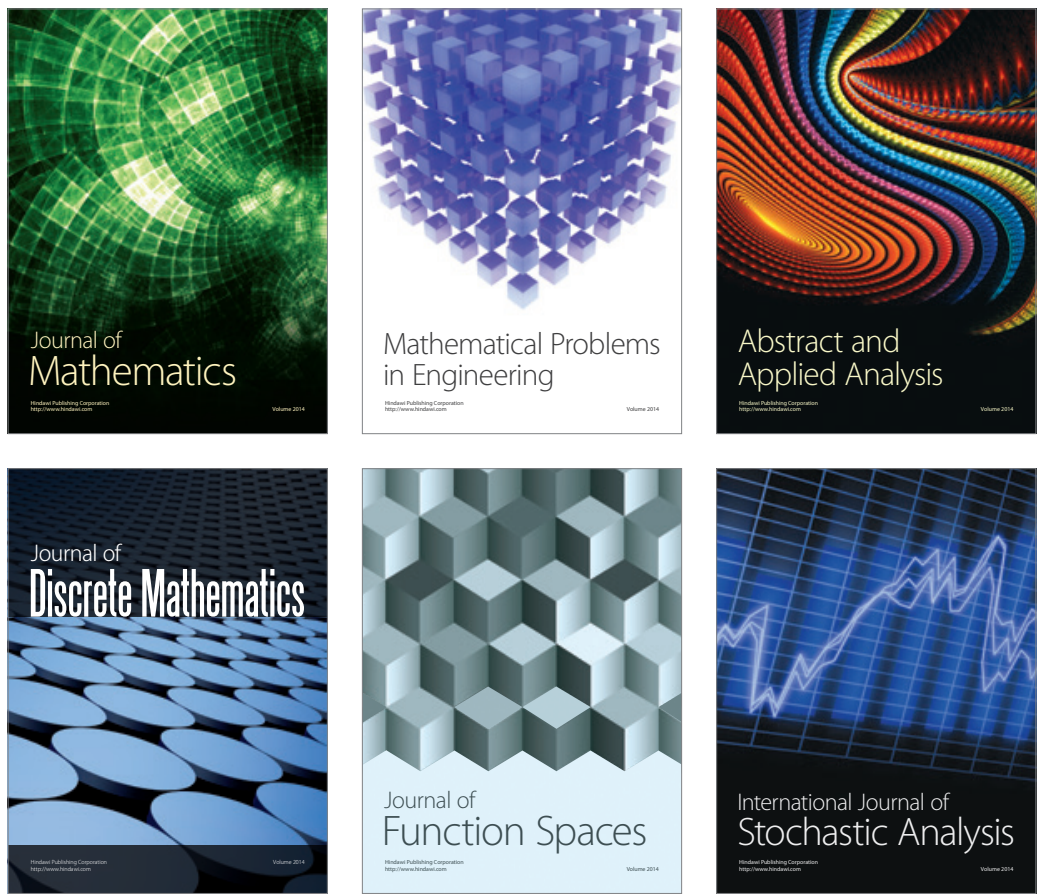

Journal of

Function Spaces

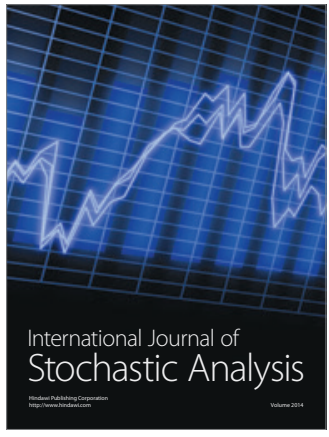

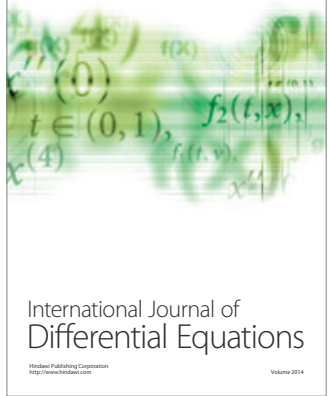
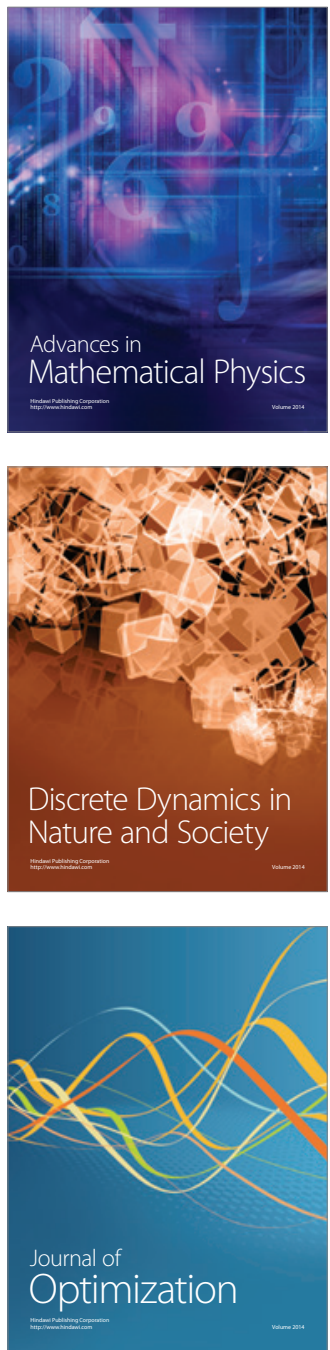